\title{
Evaluation of the Interactions Between Polymeric Chains and Surfaces with Different Structures Performed by an
} Atomic Force Microscope

\author{
Rodrigo Lambert Oréfice ${ }^{\mathrm{a}}$, Anthony Brennan ${ }^{\mathrm{b}}$ \\ ${ }^{a}$ LEPCom - Laboratório de Polímeros e Engenharia de Compósitos, \\ Departmento de Engenharia Metalúrgica e de Materiais, \\ Universidade Federal de Minas Gerais, Rua Espírito Santo 35 - $2^{\circ}$ andar, \\ 30160-030 Belo Horizonte - MG, Brazil; \\ e-mail: rorefice@demet.ufmg.br \\ ${ }^{\mathrm{b}}$ Department of Materials Science and Engineering, \\ University of Florida, 32611 Gainesville - FL, USA
}

Received: February 13, 1998; Revised: July 14, 1998

\begin{abstract}
Interactions between polymers and inorganic surfaces are present in a series of phenomena involving processes such as coagulation and deffloculation of ceramic powder and adsorption of organic macromolecules on the surface of implants, among others. In this work, Atomic Force Microscopy (AFM) was modified to allow the evaluation of interactions between polymeric chains and inorganic surfaces (silica) with different structures. Polymers (sulfonated polysulfone) were grafted onto AFM cantilevers. AFM force-distance curves were obtained for this modified tip against a series of substrates produced by depositing silica films on silicon wafers. The structure of the silica layer was modified by employing heat treatments at different temperatures. The results showed that the interactions between polymer and surfaces are dependent on the structure of the surfaces. Penetration of the polymeric chains can occur through a soft gel layer (substrates treated at low temperature, $110^{\circ} \mathrm{C}$ ). For surfaces with dense silica layers, the results showed that not only the concentration of hydroxy groups but also their spatial distribution along the surfaces are important in defining the magnitude of interactions between polymers and surfaces. A model involving a molecular recognition process, in which interactions are maximized for inorganic surfaces with structures that can match the chemical architecture of the polymer, was then used to explain the obtained results.
\end{abstract}

Keywords: AFM, polymeric chains, inorganic surfaces, adhesion, molecular recognition

\section{Introduction}

\subsection{Atomic force microscopy as a tool to investigate interactions across interfaces}

Although the importance of interfaces in a large variety of processes is well recognized, the structure of interfaces and surfaces is much less characterized than the bulk of materials. The main reasons for this lack of information are probably the combination of difficulties of searching the top layers of the material and also the usual and notorious unstable character of interfaces and surfaces. Interfaces and surfaces are very susceptible to change according to the surrounding environment, and even tough there are many analytical techniques available to investigate surfaces of materials, most of them use environments of analysis very different from the usual physical scenario applied to materials. Since surfaces are not well known, interactions between them are even more difficult to follow and description of processes often rely on analysis of the initial 
and final steps of them other than investigating the mechanisms involved.

Atomic Force Microscopy, although being a very recent technique, has rapidly spread throughout many fields of science due to its high versatility in using different types of physical and chemical aspects of surfaces and probes to give the desired output. More than allowing the processing of images on a nanometric scale, AFM has demonstrated to be very useful in investigating the magnitude of chemical and physical-chemical forces between materials on a molecular scale. Interactions between sharp AFM tips and samples can be monitored and the information obtained can be associated with physical-chemical and chemical processes, such as adhesion, van der Waals forces, electrostatic forces and even primary forces.

Since 1994, AFM has been used as a way to measure interactions involving materials. Much of the work done was related to the interactions usually involved in colloidal suspensions. The DLVO (Derjaguin-Landau-VermeyOverbeek) ${ }^{1}$ theory, which combines components due to the electrostatic repulsion and van der Waals attraction, has been fitted in AFM data, when forces between surfaces were measured. Also non-DLVO forces have been investigated such as depletion forces, hydrophobic ${ }^{2,3}$ and hydrophilic forces and others ${ }^{4}$. Surfaces forces between ceramic particles in different electrolytes, containing dissolved polymers, have been measured by $\mathrm{AFM}^{5-8}$.

Adhesion forces were also used to produce functionally dependent images ${ }^{9}$. In this case, instead of producing images from the deflection of the cantilever, images could be processed in a modified AFM by measuring the adhesion between the tip and surface and using the differences in values throughout the surface as the source of contrast.

More recently AFM has been employed to study deformation and chemical interactions between biomolecules ${ }^{10-}$ 14. The adhesion forces between individual polymeric ligand-receptor pairs were investigated by $\mathrm{AFM}^{10}$. This type of bonding is quite frequent in biological processes and is present in molecular recognition processes such as antibody-antigen and DNA complementary strands. The results showed the capability of the technique to reveal individual unbinding events ${ }^{11-14}$.

Another advantage of AFM in comparison with other analytical techniques is its ability to be used in many different types of environments, ranging from ultra-vacuum to fluids. This fact is very important for characterizing processes occurring at surfaces, since the surrounding environment at the analytical chamber can be made to mimic the real ambient conditions that define the process.

\subsection{Interactions between inorganic surfaces and} polymers

Interactions involving polymeric chains and inorganic surfaces are present in a large number of processes, including: coagulation and deffloculation of colloidal suspensions; interfacial phenomena in polymer composites; interactions between biological moieties and the surface of implants; catalytic processes induced by inorganic surfaces; and many others. It has been proposed that in most of these processes, a molecular recognition procedure between the polymeric chains and surfaces can be a critical step in determining the degree of interaction in the system. In this molecular recognition type of process, polymeric entities and species at the surfaces are continuously brought together in a attempt to minimize energies of bonding by maximizing the interaction of their chemical and structural affinities. In this picture, not only the presence of chemical and structural affinities between polymer and surfaces is important, but also how these features are distributed throughout the materials. The degree of interaction is enhanced when the spatial distribution of the mentioned features in both inorganic surface and polymer can resemble each other.

It is the purpose of this work to investigate the hypothesis that the structure of the superficial layer on inorganic substrates can regulate the interactions involving polymers and surfaces. This type of phenomenon should be present in processes such as interfacial interactions in polymerglass composites and coagulation-deffloculation processes involving polymers, among others. In glass reinforced composites, for example, the adhesion between the phases across interfaces is well known to play a fundamental role in the stress transfer process. For the experiments performed here, silica was chosen as the inorganic substrate, since the chemical structure of its surface has been extensively studied ${ }^{1,15-17}$. Presence of silanol groups $(-\mathrm{SiOH})$ is the main chemical feature of silica surfaces and the concentration of these entities is basically related to the thermal history of the material ${ }^{15}$.

In this work, interactions occurring across silica-polymer interfaces as a function of the structure of the silica surface were studied by Atomic Force Microscopy (AFM). The results were used to identify the mechanisms involved on the interfacial phenomena. Moreover, the hypothesis that interactions between a silica surface and polymer chains can be enhanced by adjusting the structure of the surface to more promptly match the chemical and structural architecture of the polymer, was also investigated. The task of reproducing the interface of the system (polymer and silica) was accomplished by preparing modified AFM tips and samples. The AFM tip was modified by incorporating a sulfonated polysulfone onto it, while silica surfaces with different nanostructures were used as the substrates for 
analysis. Sulfonated polysulfone was chosen as a probe for searching the surface of silica because it has sulfonic acid units that can readily detect structural features such as the presence of silanol groups. Interactions between this modified AFM tip and sample were then measured in an AFM liquid cell (water as solvent) and used to provide information about interactions occurring between the entities present on them. The effect of altering the structure of the silica surface on the interactions across the interface was then recorded and used to propose a model involving a molecular recognition process.

\section{Experimental}

\subsection{Preparation of modified AFM tips}

\subsubsection{Chemical modification of polysulfone}

As indicated before, polysulfone chemically modified by a sulfonation process was used as a polymeric probe of the surfaces. Chemical modification of polymers has been explored in depth for the last fifteen years. Biomedical needs motivated the modification of some polymers from hydrophobic in nature to hydrophilic by introducing polar groups on polymer backbones. Chemical modification of polymers is also used in many other applications, such as to improve chemical resistance (fluoridization of polyethylene), enhance wear resistance and others.

Polysulfone is an important material for membrane fabrication. The ability to change chemical affinities of PSF enables the production of highly selective membranes. The polysulfone repeat unit is shown in Fig. 1.

Basically, three different methods of introducing new chemical groups have been investigated to modify polysulfones: sulfonation ${ }^{18-20}$, halomethylation ${ }^{21}$ and lithiation ${ }^{22}$.

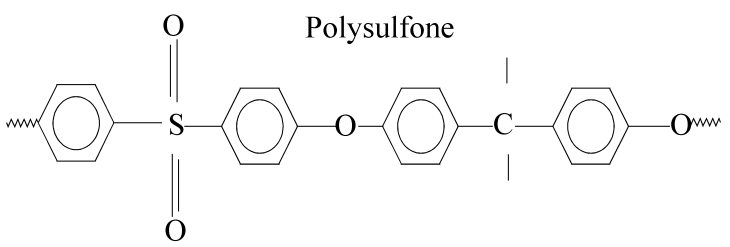

Sulfonation: chlorosulfonic acid

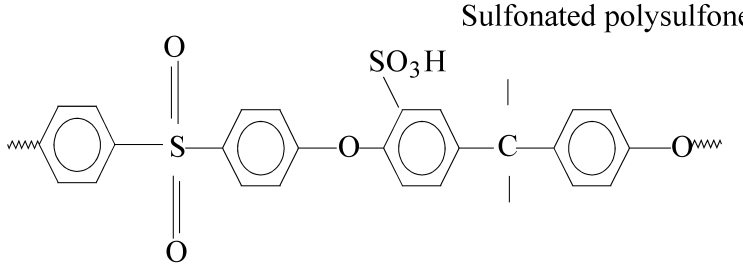

Figure 1. Sulfonation process on polysulfone.
Irradiation of the polymer with high energy sources, such as UV radiation ${ }^{23}$ have also been used to increase the hydrophilicity of polysulfone.

In this work, sulfonation of polysulfone was preferred as a way to introduce polar groups (sulfonic acid groups) on this polymer. The sulfonation of polysulfone was performed by dissolving a commercially available polysulfone (Udel®- Union Carbide, MW = 40,000 gmol) in dichloroethane (10\% wt). Chlorosulfonic acid was added to the solution at room temperature and the resultant mixture was stirred for $30 \mathrm{~min}$. The molar ratio between chlorosulfonic acid/polysulfone repeat unit was used to alter the degree of substitution.

Sulfonation of polysulfone can lead to the introduction of sulfonic acid groups $\left(-\mathrm{SO}_{3} \mathrm{H}\right)$ on the polymer. During the synthesis of the modified polysulfone, a change in the solubility behavior of the polymer could be noted and therefore evidences of the chemical modification were observed. The sulfonated polysulfone (SPSF) could readily be dissolved in solvents that are not solvents for the original polysulfone, such as ethanol and THF with $1 \%$ water, while chlorinated solvents, such as chloroform, could not dissolve the sulfonated polysulfone. A range of polymers with different degrees of substitution was produced by varying the initial concentration of chlorosulfonic acid. The effect of degree of sulfonation was readily observed regarding solubility. When high levels of sulfonation were used, water soluble sulfonated polymers could be synthesized.

Modified polysulfones were cast on KRS-5 crystals (IR transparent) and dried overnight at $60{ }^{\circ} \mathrm{C}$. Transmission FTIR (Nicolet spectrometer 20SX - 128 scans with $4 \mathrm{~cm}^{-1}$ resolution) was used to characterized the sulfonation process. The FTIR spectra of sulfonated polysulfone with different degrees of sulfonation are reproduced from 900 to $1200 \mathrm{~cm}^{-1}$ in Fig. 2. The peak at $1028 \mathrm{~cm}^{-1}$ is related to the sulfonate unit and can be correlated to the levels of substitution by ratioing it against a peak assigned to a group not involved in the modification reaction. Unfortunately, this peak overlaps the peak attributed to the ring vibration of a para-substituted aryl ether $\left(1010 \mathrm{~cm}^{-1}\right)$. In order to get the desired information from the sulfonate peak, a mathematical deconvolution procedure was performed on the peak (Fig. 3, insert). The height of the $1028 \mathrm{~cm}^{-1}$ peaks was then ratioed against the $1010 \mathrm{~cm}^{-1}$ peaks. The degree of substitution of a specific sulfonated polysulfone was calculated using NMR data published in another manuscript ${ }^{24}$. This value was used to calibrate the FTIR data of polymers with different degrees of substitution, by associating it to the FTIR peak ratio of the same polymer. A plot of the effect of the initial ratio between reactants (polysulfone repeat unit and chlorosulfonic acid) on the degree of substitution, calculated as indicated above is shown in Fig. 3. Polymers with different levels of sulfonation could therefore be synthesized by changing the initial stoichiometry of the reac- 
tion. A scheme of the reaction procedure is shown in Fig. 1. The NMR study ${ }^{24}$ also demonstrated that the substitution occurs preferentially on the ortho position, in relation to the ether group, of benzene rings at the Bisphenol A unit (Fig. 1).

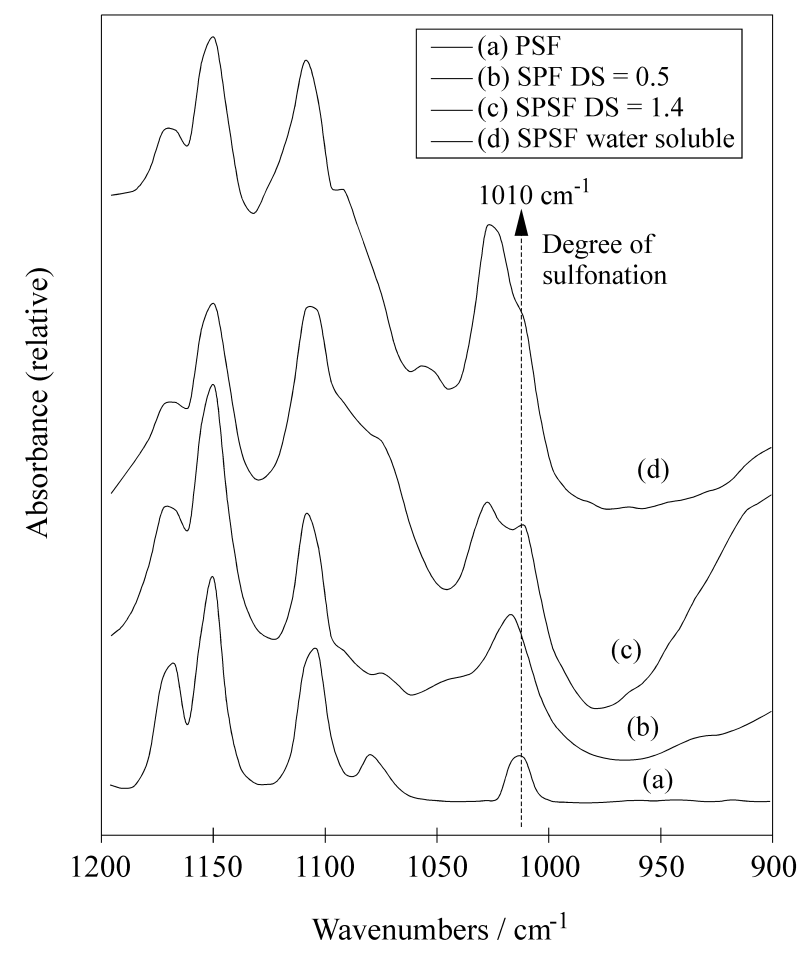
of substitution

FTIR spectra of sulfonated polysulfones with different degrees

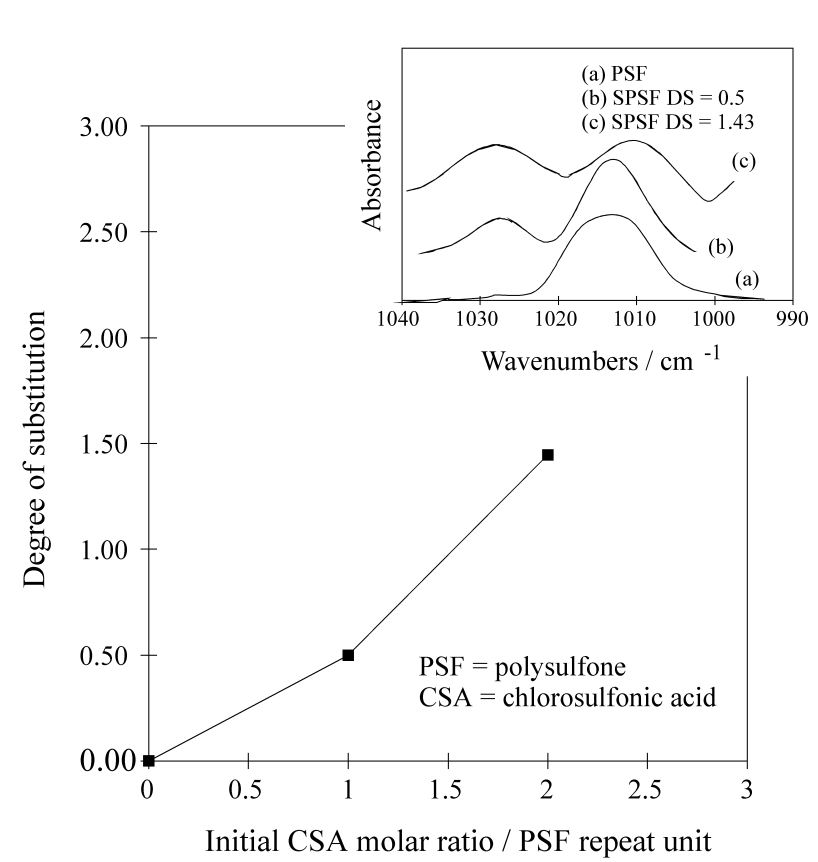

Figure 3.

of polysulfone. Top of the figure: deconvolution of FTIR spectra used to determine the degree of sulfonation.
2.1.2. Grafting modified polysulfones on the surface of glass spheres

In order to simulate the interface proposed (polymer against silica), a conventional AFM tip was modified by attaching a glass sphere (Polyscience, $15 \mu \mathrm{m}$ radius) to the cantilever. Before attaching it to the cantilever, sulfonated polysulfone, with a low degree of substitution (0.5), was grafted onto the surface of the spheres. In order to graft the sulfonated polymer on the spheres, their surfaces were firstly treated with aminopropyl triethoxysilane (APS) by dispersing the spheres in an ethanol-water-APS for one hour and curing them at $110{ }^{\circ} \mathrm{C}$ for $12 \mathrm{~h}$. The APS treated spheres were then reacted with SPSF (sulfonated polysulfone) via formation of sulfonamide bonds, by dispersing them in a SPSF-dimethylformamide solution for $2 \mathrm{~h}$. The spheres were collected by filtration, dried overnight at $120{ }^{\circ} \mathrm{C}$ under vacuum and washed extensively with dimethylformamide, to remove any non-bonded polymer.

To verify the success of the grafting technique and to determine the capabilities of the new polymers to bond via primary bond formation to the silane treated glass spheres, X-ray photoelectron spectroscopy (XPS) was performed on a soda-lime silicate glass disk containing grafts of the modified polysulfone. X-ray photoelectron spectroscopy was performed in a XPS, Kratos DS800 Mg radiation, system. A $90^{\circ}$ incident beam was used and the results were corrected using the carbon photoelectron peak at $285 \mathrm{eV}$. Prior to the analysis, the samples were dried at $90{ }^{\circ} \mathrm{C}$ and reduced pressure for $12 \mathrm{~h}$.

For the XPS measurements, glass disks $(1 \mathrm{~cm}$ in diameter), with composition close to the composition of the glass spheres (glass type E), were cut and polished with a $\mathrm{SiC}$ sand paper \# 600. Disks were treated with a silane coupling agent (aminopropyl triethoxysilane) by introducing them into a solution of amino silane (1 vol.\%) in ethanol-water (95 vol.\% ethanol) for one hour. The treated disks were then cured at $110^{\circ} \mathrm{C}$ for 1 day. After this, the samples were introduced into a solution of sulfonated polysulfone in ethanol ( $5 \mathrm{wt} \%)$ for $2 \mathrm{~h}$. Subsequently, disks were washed several times with ethanol and any non-bonded specie was removed by using a Sohlex extractor for $12 \mathrm{~h}$.

XPS data was collected in Perkin-Elmer spectrometer. The XPS spectra (from 0 to $600 \mathrm{eV}$ ) of a glass disk and a glass disk with grafted sulfonated polysulfone are reproduced in Fig. 4. The peaks identified in the spectrum of the glass disk with sulfonated PSF grafts were mainly related to $\mathrm{C}(1 \mathrm{~s}), \mathrm{S}(2 \mathrm{p})$ and $\mathrm{O}(1 \mathrm{~s})$. A semi-quantitative software routine, that computes the area under the peaks, was used to verify the amount of each element on the surface of the sample. The theoretical composition of the sulfonated PSF was calculated using the values of degree of substitution given by the NMR/FTIR studies. These calculations (Table 1) demonstrated that a large part of the surface was covered 
Table 1. Comparison between theoretical composition and results obtained from XPS of glass surfaces grafted with modified polysulfone.

\begin{tabular}{lccc}
\hline Elements & PSF* $^{*}$ wt. \%) & Sulfonated PSF* (wt. \%) & Grafting SPSF** onto glass (wt. \%) \\
\hline $\mathrm{C}(1 \mathrm{~s})$ & 77.1 & 62 & 61.67 \\
$\mathrm{O}(1 \mathrm{~s})$ & 15.3 & 24 & 23.13 \\
$\mathrm{~S}(2 \mathrm{p})$ & 7.6 & 14 & 13.34 \\
$\mathrm{~N}(1 \mathrm{~s})$ & - & - & - \\
$\mathrm{Ca}(2 \mathrm{p})$ & - & - & 1.82 \\
$\mathrm{Si}(2 \mathrm{p})$ & - & & - \\
\hline
\end{tabular}

* Theoretical values.

** SPSF $=$ sulfonated polysulfone.

with the sulfonated polysulfone. Very little signal coming from elements typically in the glass could be captured, such as silicon and calcium. Another important observation from these results is that the theoretical composition of sulfonated polysulfone is very close to the obtained results. Therefore, the success of the grafting procedure was proven. Moreover, the new polymer was grafted onto the surface of glass possibly through covalent bonds between sulfonic acid groups and amine groups on the silane layer (formation of sulfonamide bonds $-\mathrm{SO}_{2}-\mathrm{NH}-$ ).

\subsection{Attachment of modified glass spheres onto AFM cantilevers}

The modified glass spheres (with sulfonated polysulfone grafts) were attached onto AFM silicon nitride cantilevers by using a procedure similar to the one reported by Duker $^{17}$. An epoxy (DGEBA - dyglicidyl ester biphenol A) with a diamine hardener was used to glue the sphere to the cantilever. The epoxy and sphere were transferred to the cantilever using a micromanipulator coupled with an optical microscope. The epoxy was cured overnight at room temperature. Figure 5 shows a SEM micrograph of a silicon nitride AFM tip that has four cantilevers, two in each side. In Fig. 5, one modified cantilever (SPSF treated glass sphere) and a non-modified one are shown. Figure 6 shows the procedure involved on the preparation of modified AFM tips.

\subsection{Preparation of Silica Substrates}

The substrate chosen to accommodate the silica surfaces in the AFM studies was a silicon wafer. Silicon wafers were firstly cleaned with acetone and dichloroethane and then submitted to a heat treatment at $420{ }^{\circ} \mathrm{C}$ for one hour in air to develop a thin silica layer at the top of the silicon wafer. Hydroxylation of the newly formed silica layer was performed by dipping the sample in boiling water for $6 \mathrm{~h}$. In order to control the nanostructure of the oxide layer, a thin silica-gel layer was deposited on the surface of the grown oxide by using the sol-gel process. Silicon wafers were dipped into a tetraethoxy silane (TEOS)/etha-

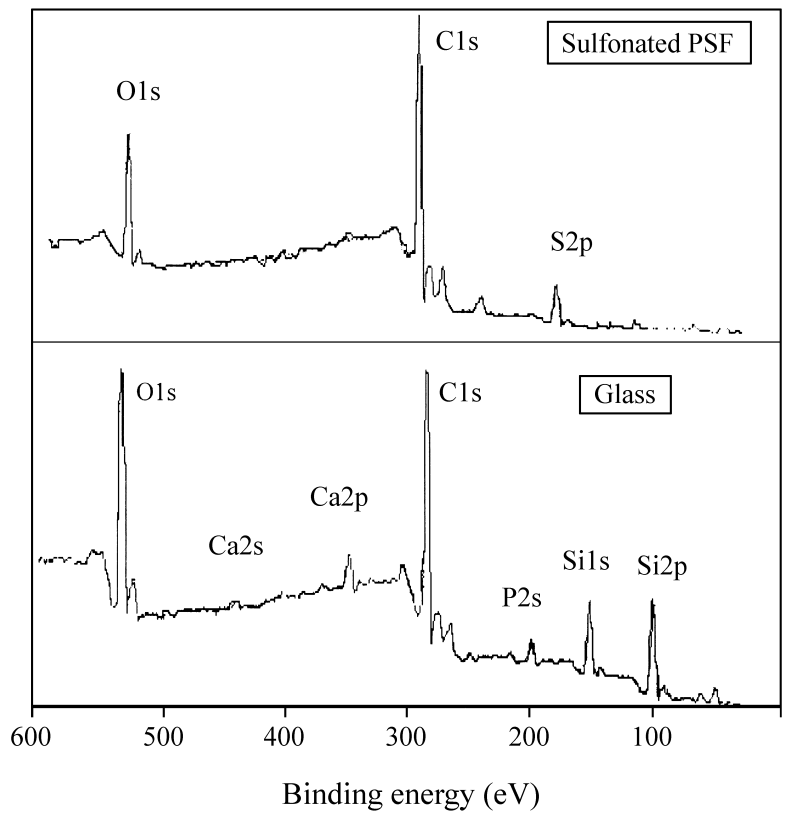

Figure 4. XPS spectra of glass samples with grafted polysulfone.

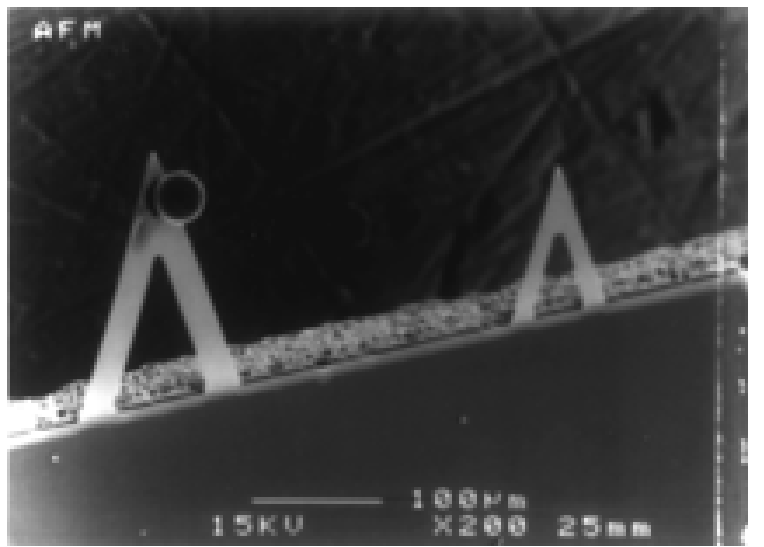

Figure 5. SEM micrograph of a glass sphere attached to an AFM cantilever.

nol/water solution ( $1 \%$ vol. TEOS, $\mathrm{pH}=2$ ) for one hour. The nanostructure of the silica layer was changed by heat treating the substrates at different temperatures: 110, 400, 
600 and $800{ }^{\circ} \mathrm{C}$. It is known that heat treatments on silica gels can change structural features of the material, such as: (1) reduce the concentration of silanol groups; (2) reduce surface area; (3) increase number of siloxane bonds, i.e., the cross-link density; (4) increase both bulk and structural density; (5) shift the distribution of silica rings to larger number of tetrahedra.

\subsection{Measurements}

A Nanoscope III (Digital Inst., Santa Clara, CA) was used in contact mode. In order to avoid the formation of a thin liquid film on the substrate that usually adds a capillary force component to the measured forces by AFM, a fluid cell accessory, provided also by the manufacturer, was used. Deionized water $(\mathrm{pH}=5.0)$ was used during the measurements. Water is not a good solvent for the sulfonated polysulfone used (low degree of substitution), but swell the polymer.

Force-distance measurements were performed by applying to the AFM piezoelectric a saw-tooth type of curve. In this case, instead of keeping the height or force constant, as usual when images are being processed in contact mode, the piezoelectric will move the sample against the tip in the vertical direction ( $\mathrm{z}$ direction) in a cyclic way. In each cycle, the deflection of the cantilever is recorded as a function of the travel of the piezoelectric. When the tip is approaching the surface of the sample, an attractive force is usually present, represented by any one of the type of chemical forces (electrostatic, van der Waals, etc.). A "jump" of the tip towards the sample surface occurs when the elastic constant of the cantilever cannot keep up with the gradient of forces acting on the tip. When an initially in-contact tip is released from the surface, another characteristic jump is produced in which the tip sticks to the surface and an extra force is required to unload the tip. The magnitude of this force can be related to the adhesion between the materials on the tip and sample. The raw data provided by the equipment is usually given in a curve where the deflection of the cantilever is plotted against the distance traveled by the piezoelectric. In order to convert this raw data to the force-distance curve, positions of zero force and zero displacement need to be assigned. Zero forces are defined where the cantilever is very far from the sample, and therefore no forces are present. The zero displacement is assigned to the position where the repulsive forces acting on the cantilever start to display a linear behavior, meaning that the tip is elastically compressing the sample. The parameters of the technique usually used to manipulate the way the tip and sample will come together are: z-scan start, that is basically the initial height from where it starts the approach; z-scan size, that is the total distance traveled by the piezoelectric during one extension-retraction cycle; z-scan rate, that is the rate of oscillation. By making use of those parameters, it is possible to perform measurements far from the surface or close to the surface with different amplitudes of oscillation. Unless otherwise specified, scan rate of $1 \mathrm{~Hz}$ and scan size of $500 \mathrm{~nm}$ were used throughout this work. Many sites in each sample were used to obtain the force-displacement curves, thus ensuring reproducibility of results.

In order to ensure that the procedures of attaching the glass sphere to the cantilever and measuring the AFM forces were being employed successfully, a special tip was prepared by attaching a plain glass sphere on the cantilever. The interactions between this tip and the silicon wafer sample treated at $110^{\circ} \mathrm{C}$ were measured in water $(\mathrm{pH}=5)$. The raw data as well as the converted data are shown in Fig. 7. The basic result is that there is no attractive forces during tip extension and no adhesive forces during tip retraction. These results are similar to the ones reported by others ${ }^{1,17}$ for a silica-silica system. At $\mathrm{pH} 5$, silica surfaces are negatively charged and electrostatic repulsion will occur. Moreover, hydration forces (where the water molecules are oriented nearby the surface) are usually employed to explain the short range repulsive forces that appear in a silica system also in $\mathrm{pH}$ 's closer to the isoelectric point of this material. The presence of silicate chains at the surface has also been used to explain the phenomenon ${ }^{1}$.

\section{Discussion}

In Fig. 6, a drawing representing the whole technique developed to measure interactions between surfaces and polymeric entities grafted on modified AFM tips (glass sphere with polymer grafts) is shown. In this scenario, the polymer chains are being brought continuously in and out of contact with the surface. This cyclic mechanism mimics

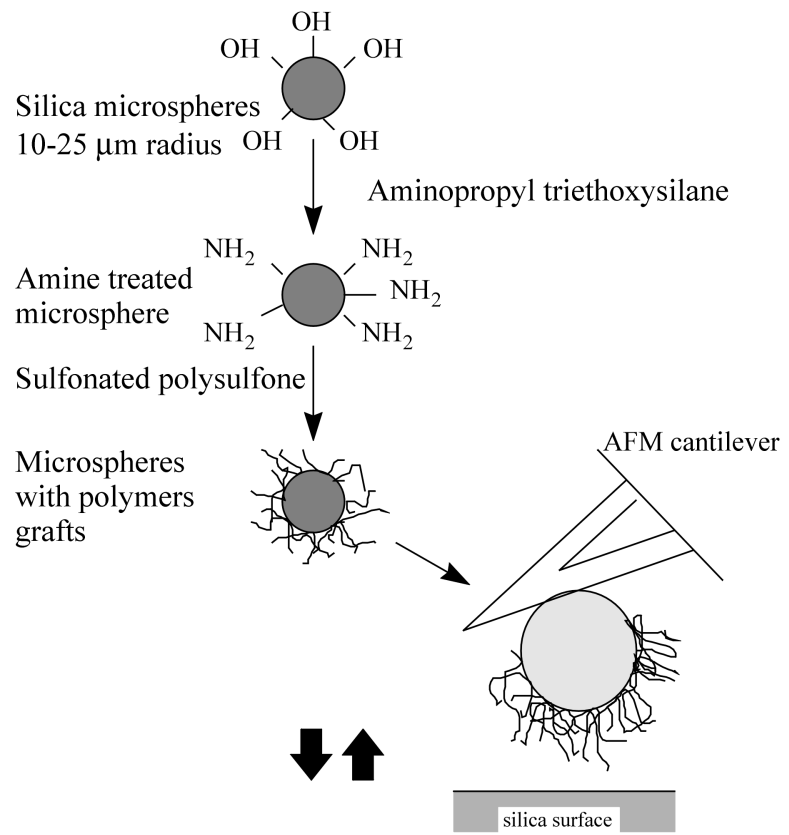

Figure 6. AFM tip preparation and measurements. 
natural processes where polymeric chains hit the surface of substrates looking for positions with lower energy to adsorb. As show in this figure (Fig. 6), the use of a sulfonated polysulfone with a low degree of substitution favors the formation of few linkages between the polymer and the surface of the glass sphere during the tip preparation, thus allowing the presence of fairly large non-bonded chain lengths.

The results of force-distance curves obtained by AFM in aqueous solution $(\mathrm{pH}=5.0)$ are shown in Fig. 8 for a glass sphere treated with sulfonated polysulfone (SPSF) in contact with silica substrates. For this experiment, the same tip was used in all the measurements, and therefore it was possible to use the average value of the cantilever elastic constant (provided by the manufacturer, $\mathrm{k}=0.12 \mathrm{~N} / \mathrm{m}$ ) on the calculations (conversion of deflection in force). At the end of the measurements, the first sample investigated was again tested to ensure that no major degradation of the polymer grafts had occurred during previous measurements.

A typical force-distance curve for the interaction between a sphere treated with SPSF and the silica substrate heat treated at $800{ }^{\circ} \mathrm{C}$ can be seen in Fig. 8. In this figure, the tip extending toward the surface and coming out of the surface are observed respectively in Figs. 8-e and 8-d. It is important to realize the differences between this curve and the curve in Fig. 7, where a plain sphere was used instead of the SPSF treated sphere. In the case of the polymer grafted sphere, an attractive jump can be noted as well as a large adhesive force during tip retraction, while only repulsion was measured for the pure glass sphere. This result proves that the systems are very different, suggesting the presence of polymer on the surface of the sphere and confirming the XPS results.

Although the ionization equilibrium of sulfonated polysulfone is not known, it is very likely, based on the behavior of other sulfonated materials, that sulfonated polysulfone should have some of its sulfonic acid groups protonated at $\mathrm{pH}$ 's close to 5.0. Therefore sulfonated PSF can exhibit positive charges in some locations. These positive charges located far from the surface can then interact electrostatically with the approaching negatively charged silica surface, leading to the attractive jump seen in Fig. 8-e. After being compressed onto the silica surface during tip extension, many more groups on the polymer will interact strongly with the silica surface not only by electrostatic forces, but mainly by hydrogen forces between silanol groups and sulfonic acid groups.

The retracting path of the tip, shown for all the substrates in Fig. 8, reveals that the adhesion of the polymer to the silica sample is a function of the silica structure. A maximum AFM adhesion was obtained for the silica sample treated at $110^{\circ} \mathrm{C}$. At this temperature $\left(110^{\circ} \mathrm{C}\right)$, the silica top layer is fully hydrated and has an open and less packed structure with lower cross-link density. This situation facilitates penetration of free chain ends through the open network, maximizing chemical interactions (hydrogen bonds between silanol groups and sulfonic acid groups) and entanglements. Figure 9 plots the effect of the temperature

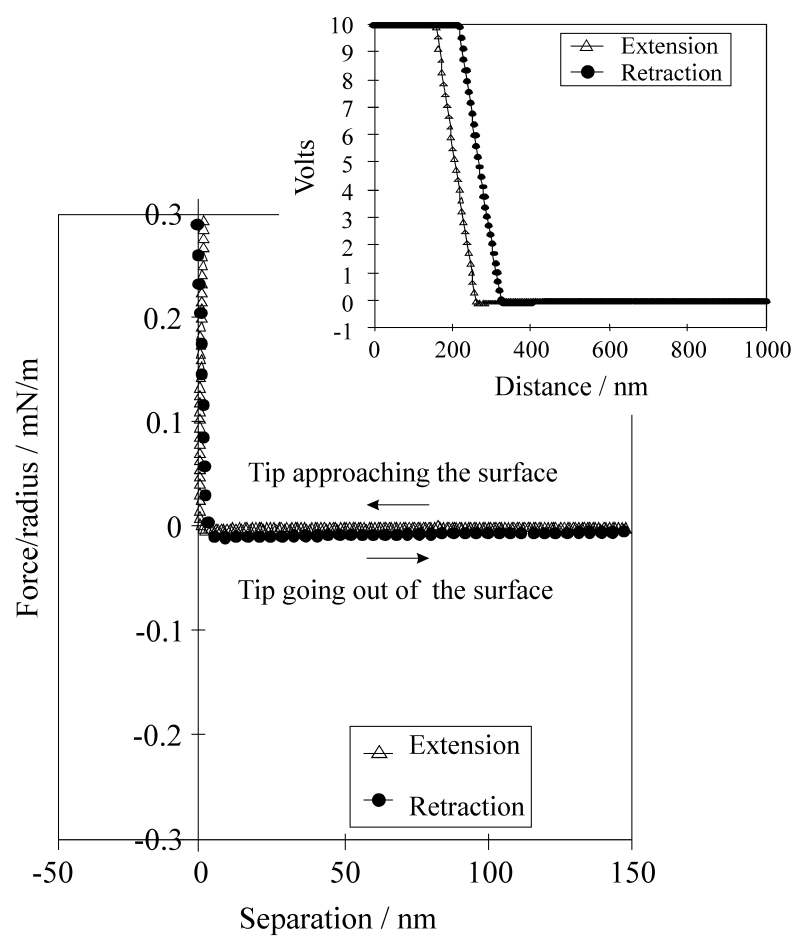

Figure 7. Force-distance curve for a glass sphere attached to an AFM cantilever against a silica surface in water. Raw data shown on the top.

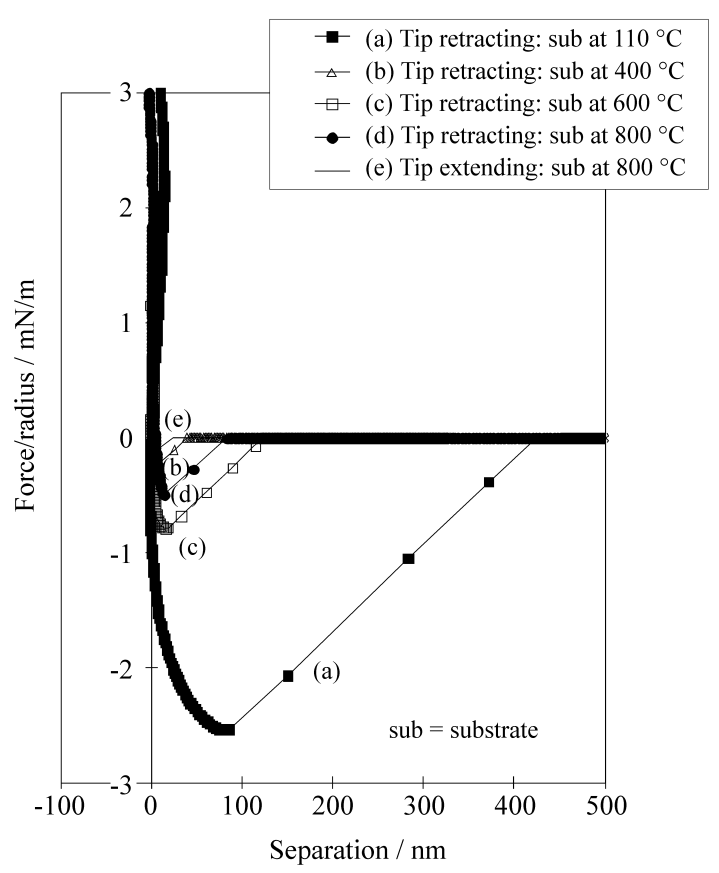

Figure 8. AFM force-distance curves for a sulfonated polysulfone tip and silica surfaces treated at different temperatures. 


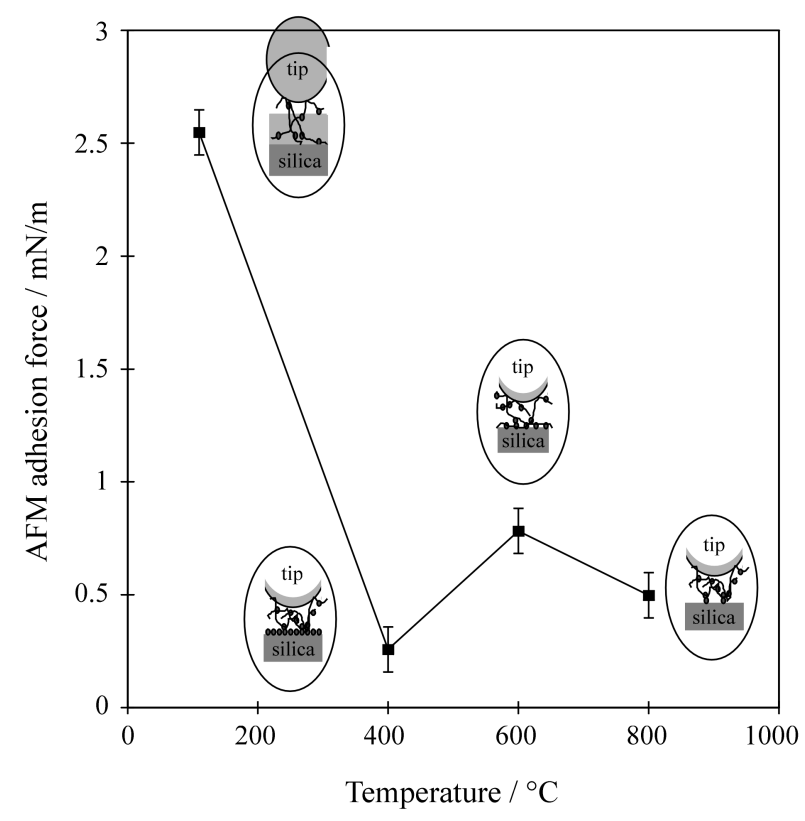

Figure 9. Effect of the temperature of heat treatment of silica surfaces on the AFM adhesion of a sulfonated polysulfone tip. Drawings represent the mechanisms used to explain the results ( filled circles $=\mathrm{OH}$ groups).

of heat treatment of the substrates on the adhesion measured by AFM.

As it can be observed in Figs. 8 and 9, a minimum value of adhesion was measured for the substrate treated at $400{ }^{\circ} \mathrm{C}$. At $400{ }^{\circ} \mathrm{C}$, the surface of silica retains a large concentration of silanol groups ${ }^{15}$, but the network is much more dense due to the progressing of condensation reactions during the thermal treatment.

On the other hand, higher levels of adhesion were measured for interactions between the tip and substrates treated at 600 and $800{ }^{\circ} \mathrm{C}$. From $400{ }^{\circ} \mathrm{C}$ to $600{ }^{\circ} \mathrm{C}$, silanol groups are removed from the surface and, thus, isolation of silanol groups occurs. From $600{ }^{\circ} \mathrm{C}$ to $800^{\circ} \mathrm{C}$, the isolated silanol groups are progressively removed. Therefore, it is proposed that the presence of isolated silanol groups allows higher levels of interactions between a polymer (intrinsically capable of interacting with silanol groups via hydrogen bonds) and silica than a highly hydrated surface. In a highly hydrated silica surface, the overlapping of chemical potential fields of nearby silanol groups restricts the adsorption of the polymer by reducing the number of conformational modes that the polymer can have on the surface. Only conformational modes that can limit the interaction between hydrophobic groups on the polymer and silanol groups should be able to contribute to adhesion.

The fundamental result obtained from the AFM study was that the nanostructure of the silica surface can definitely regulate interactions between species present on the interface of polymer-inorganic material systems and there- fore can define interfacial properties, such as adhesion and others.

Based upon the AFM results, it can be proposed that two different group of mechanisms of interactions between polymer chains and silica surface were detected. The first one occurs when polymer chain interact with a less packed, low density and soft silica-gel layer. In this case, penetration of the chains through this gel layer is allowed. Large degrees of adhesion are obtained due basically to both mechanical interlock of chains inside the gel network as well as to chemical interactions between silanol groups and sulfonic acid groups on the polymer. This situation is illustrated in the drawings shown in Fig. 9. This type of mechanism is used to explain adhesion between polymer chains and silane coated reinforcing agents in polymer composites $^{24,25,26}$.

When the gel layer is heat treated at higher temperatures (above $400{ }^{\circ} \mathrm{C}$ ), densification of the network leads to an increase in the bulk density, reduction in the surface area and an increase in the elastic modulus of the layer. As a consequence, penetration of the polymeric chains through the network is restricted and interactions will basically resume to chemical bonding between silanol groups on the surface and sulfonic acid groups on the polymer (second group of mechanisms detected), via formation of hydrogen bonds. In this case, adhesion between polymeric chains and the surface is improved when the number of silanol-sulfonic acid linkages per unit area is maximized. Although the opportunities for a silanol-sulfonic acid bond to occur should be maximum for a surface filled with surface silanol groups (surface at $400^{\circ} \mathrm{C}$ ), this situation can also maximize the probability of a less energetically possible interaction between hydrophobic units of polysulfone (benzene rings, for example) and silanol groups. Conformation modes of the polymeric chains that promote this type of interaction are forbidden, leading to a reduction in the total number of conformation modes and consequently a reduction in entropy and an increase in free energy for bonding. The overall result of this phenomenon is a reduction in adhesion between the surface and polymer.

When the silica surface is submitted to temperatures above $400{ }^{\circ} \mathrm{C}$, silanol groups on the surface are progressively eliminated and become isolated. This process will allow the formation of a chemically patterned surface, where chemical functionalities, such as silanol groups, are equally spaced on average from each other. The isolation of silanol groups will allow conformation modes of the polymer chains, forbidden on substrates treated at $400{ }^{\circ} \mathrm{C}$, to exist by restricting interactions between hydrophobic units on the polymer and hydrophilic silanol groups. This phenomenon will eventually lead to a situation where the spatial arrangement of silanol groups on the surface of the silica substrate can match the distribution pattern of the sulfonic acid groups on the polymer structure. In this case, 
the inorganic surface acts as a template for the interaction of polymeric chains, leading to a maximized adhesion. Figure 9 also illustrates this phenomenon.

From the discussion above, it is possible to propose that a molecular recognition phenomenon can regulate interactions between polymers and patterned surfaces. Based upon the obtained results, it can be proposed that a series of processes can be improved by either adapting the chemical architecture of the polymers to a specific surface (such as in the case of coagulation of ceramic powder) or designing structures of surfaces to interact in an optimized way with polymeric chains. In the biomaterial field of science, for example, the structure of the surface of implants has to be designed to either favor protein and cell adhesion or reduce it. In the case of intraocular lens (for the treatment of cataracts), it was observed that lens with highly hydrophobic or hydrophilic surfaces can reduce cell adhesion (that is aimed), while surfaces with an intermediary character favors protein and cell adhesion ${ }^{27}$. Based upon the results of this work, it is clear that the combination of hydrophobic and hydrophilic groups on the surface of the implant can possibly match the sequence of distribution of hydrophobic and hydrophilic groups on polymeric biomolecules, leading to high levels of adhesion.

\section{Conclusion}

In this work, interactions between polymeric entities and the surface of inorganic materials were monitored by using an Atomic Force Microscope (AFM).

The interface between polymers and inorganic surfaces was mimic by modifying an AFM tip and using this tip to investigate the surface of a series of silica substrates with different nanostructures. The modification of AFM tips was accomplished by grafting sulfonated polysulfone onto it. Sulfonated polysulfone was prepared by sulfonating a commercial polysulfone. The newly introduced sulfonic acid groups on polysulfone can examine the surface of the silica substrate by interacting with silanol groups.

The results of this work revealed that AFM can be an important tool to investigate phenomena occurring at the interfaces of materials and systems. In this work, mechanisms involved in the interfacial phenomena, such as chain penetration, adsorption, chemical bond formation, entanglements could be studied using the developed AFM procedure.

The results of the AFM force-displacement curves showed that adhesion of the polymeric chains to the surface is a function of the structure of the surface. It was proposed that penetration of polymer chains through surfaces with low cross-link density and high porosity was responsible for the high levels of interactions measured. For dense surfaces, the interactions between polymer and surfaces were shown to be dependent on both the concentration and spatial distribution of silanol groups on the surface. A molecular recognition type of process, in which interactions between the phases are enhanced for structures of surfaces that matches the chemical architecture of the polymer, was proposed to be responsible for the obtained results.

\section{Acknowledgment}

The authors gratefully acknowledge the financial assistance of CNPq (Conselho Nacional de Desenvolvimento Científico e Tecnológico) of the Brazilian Government and the NIH (National Institutes of Health) of the Government of the United States, grant \# DEO 9307-07.

\section{References}

1. Israelachvili, J.N. Intermolecular and Surface Forces, Academic Press, San Diego, 1991.

2. Mantel, M.; Rabinovich, J.P.; Wightman, J.P.; Yonn, R.-H. A study of hydrophobic interactions between stainless steel and silanated glass surface using atomic force microscopy. J. Colloid Interface Sci., v. 170, p. 203-14, 1995.

3. Rabinovich, Y.I.; Yoon, R.-Y. Use of atomic force microscopy for the measurements of hydrophobic forces between silanated silica plate and glass sphere. Langmuir, v. 10, p. 1903-9, 1994.

4. Ackler, H.D.; French, R.H.; Chiang, Y.M. Comparisons of Hamaker constants for ceramic systems with intervening vacuum or water from force laws and physical-properties. J. Colloid Interface Sci., v. 179, p. 460-469, 1996.

5. Toikka, G.; Hayes, R.A.; Ralston, J. Surface forces between spherical $\mathrm{ZnS}$ particles in aqueous-electrolyte. Langmuir, v. 12, p. 3783-3788, 1996.

6. Biggs, S. Steric and bridging forces between Surfaces bearing adsorbed polymer. An atomic force microscopy study. Langmuir, v. 11, p.156-162, 1995.

7. Biggs, S. Nonequilibrium interaction forces between adsorbed polymer layers. J. Chem. Soc.-Faraday Trans., v. 92, p. 2783-2789, 1996.

8. O'Shea, S.J.; Welland, M.E.; Rayment, T. An atomic force microscope study of grafted polymers on mica. Langmuir, v. 9, p. 1826-35, 1993.

9. Berger, C.E.H.; Vanderwerf, K.O.; Kooyman,R.P.H.; Degrooth, B.G.; Greve, J. Functional-group imaging by adhesion afm applied to lipid monolayers. Langmuir, v. 11, p. 4188-4192, 1995.

10. Florin, E-L.; Moy, V.T.; Gaub, H.E. Adhesion forces between individual ligand-receptors pairs. Science, v. 264, p. 415-7, 1994.

11. Moy, V.T.; Florin, E-L.; Gaub, H.E. Intermolecular forces and energies between ligands and receptors. Science, v. 266, p. 257-9, 1994. 
12. Grubmüller, H.; Heymann, B.; Tavan, P. Ligand binding: molecular mechanics calculation of the streptavidin-biotin rupture force. Science, v. 271, p. 997-9, 1996.

13. Lee, G.U.; Chrisey, L.A.; Colton, R.J. Direct measurement of the forces between complementary strands of DNA. Science, v. 266, p. 771-3, 1994.

14. Rief, M.; Oesterhelt, F.; Heymann, B.; Gaub, H.E. Single molecule force spectroscopy on polysaccharides by atomic force microscopy. Science, v. 275, p. 1295-7, 1997.

15. Zhuravlev, L.T. Concentration of hydroxyl groups on the surface of amorphous silicas. Langmuir, v. 3, p. 316-8, 1987.

16. Vigil, G.; Xu, Z.; Steinberg, S.; Israelachvile, J. Interactions of silica surfaces. J. Colloid Interface Sci., v. 165, p. 367-385, 1994.

17. Ducker, W.A.; Senden, T.J.; Pashley, R.M. Measurement of forces in liquids using a force microscope. Langmuir, v. 8 (1992), 1831-6.

18. Brousse, C.L.; Chapurlat, R.; Quentin, J.P. New membranes for Reverse Osmosis 1. Characteristics of the base polymer: sulphonated polysulphones. Desalination, v. 18, p. 137, 1976.

19. Pozniak, G.; Krajewska, B.; Trochimczuk, W. Urease immobilized on modified polysulphone membrane: Preparation and properties. Biomaterials, v. 16, p. 129, 1995.

20. Chen, M.; Chiao, T.; Tseng, T. Preparation of sulfonated polysulfone/polysulfone and aminated polysul- fone/polysulfone blend membranes. J. Appl. Polym. Sci., v. 6, p. 1205-1209, 1996.

21. Higuchi, A.; Koga, H.; Nakagawa, T. Surface-modified polysulfone hollow fibers. IV. Chloromethylated fibers and their derivatives. J. Appl. Pol. Sci., v. 46, p. 449, 1992.

22. Guiver, M.D.; Robertson, G.P. Chemical Modification of Polysulfones: An facile Method of Preparing Azide Derivatives from Lithiated Polysulfone Intermediates. Macromolecules, v. 28, p. 294, 1995.

23. Nyström, M.; Jaervinen, P. Modification of polysulfone ultrafiltration membranes with UV irradiation and hydrophilicity increasing agents. J. Membr. Sci. v 60, p. 275-296, 1991.

24. Oréfice, R.L.; Brennan, A.B. An Atomic Force Microcopy study of the interactions involving polymers and silane networks. Submitted for publication in Revista Polímeros: Ciência e Tecnologia, 1998.

25. Suzuki, N.; Ishida, H. A review on the structure and characterization techniques of silane/matrix interphases. Macromol. Sym., v. 108, p. 19-53, 1996.

26. Schutte, C.L. Environmental durability of glass-fiber composites. Mat. Sci. Eng. R-reports, v. 13, p. 265323, 1994.

27. Werner, L.P.; Legeais, J.M.; Durand, J.D.; Savoldelli, M.; Legeay, G.; Renard, G. Endothelial damage caused by uncoated and fluorocarbon- coated poly(methyl methacrylate) intraocular lens. J. Caract. Refract. Surg. v. 23, p.1013-9, 1997. 